\title{
Depth without disparity in random-dot stereograms
}

\author{
ROBERT P. O'SHEA and RANDOLPH BLAKE \\ Cresap Neuroscience Laboratory, Northwestern University, Evanston, Illinois
}

\begin{abstract}
Depth can be perceived in random-dot stereograms in which dots are binocularly uncorrelated, in the absence of overall positional disparity (e.g., Julesz, 1960). This phenomenon, which we have called rivaldepth, persists over a wide range of luminances, stereogram dot densities, and dot sizes. Rivaldepth is also observed with interocular uncorrelation provided by complementation and orthogonal rival contours, and in both static and dynamic random-dot stereograms. Like conventional stereopsis, rivaldepth magnitude grows with increasing viewing distance, but unlike conventional stereopsis its direction does not reverse when half-images are interchanged. In a survey of 52 individuals, about half consistently reported far rivaldepth; they were also more likely to exhibit overconvergence on a test of fixation disparity. The remaining subjects reported near rivaldepth and tended to show underconvergences. A second experiment, however, showed that eye movements measured immediately after offset of rivaldepth stereograms were in the opposite direction (e.g., convergences were associated with near depth). Evidently, once rivaldepth is established, subjects attempt to fuse the "object" projecting the uncorrelated images. Rivaldepth magnitude is similar to depth produced by stereograms with disparities too large to be fused. The pattern of eye movements and rivaldepth magnitude suggest that the binocular visual system treats uncorrelation as a potentially correlated disparity beyond the fusion limit.
\end{abstract}

It has been known for over a century (Wheatstone, 1838) that stereoscopic depth can be produced when the two eyes view displays in which matching portions of the two monocular views fall on noncorresponding retinal areas (i.e., a positional disparity exists between portions of the left- and right-eye views). Perhaps the most dramatic demonstration of the effectiveness of positional disparity is provided by random-dot stereograms, in which depth is signaled by some consistent disparity among locally identical elements (Julesz, 1971).

It is not well known, however, that stereoscopic depth can also be elicited in the absence of consistent positional disparity. In particular, when random-dot stereograms containing relatively large regions in which dots are uncorrelated interocularly are viewed, the uncorrelated regions, besides yielding patent binocular rivalry, produce stereoscopic depth in the absence of overall horizontal disparity. For purposes of discussion, we shall term this percept rivaldepth. From the outset, we should stress that this phenomenon is not novel. Other investigators, most notably Julesz (1960), have described comparable depth effects. In the General Discussion, we shall relate

\footnotetext{
This research was supported by NSF Grant BNS 8418731. Preliminary results were presented to the Annual Meeting of the Association for Research in Vision and Ophthalmology, Sarasota, Florida, April 28-May 2, 1986. We thank Gian Poggio and Oliver Braddick for sharing the results of their unpublished research. We are particularly grateful to Robert Patterson and Karen Holopigian for comments on an earlier version of this manuscript. Address reprint requests to Robert $O$ 'Shea at Cresap Neuroscience Laboratory, Northwestern University, 2021 Sheridan Rd., Evanston, IL 60201.
}

those earlier observations to the ones investigated in this paper.

Our interest in this phenomenon originated from a related study concerned with depth in conventional, fully correlated random-dot stereograms containing a central region whose retinal disparity was too large to be fused. To our surprise, the depth associated with these large disparities was sometimes opposite that predicted from the sign of the disparity. For instance, large crossed disparities tended to be seen as far depth. For these initial observations, several naive observers were asked simply to estimate the magnitude of depth of the central region in inches and to report on its appearance. The stereograms consisted of a matrix of $240 \times 240$ dots, with a central square region consisting of $110 \times 110$ dots; each dot had an equal probability of being black or white. Between disparities of $0.5^{\circ}$ uncrossed and $0.5^{\circ}$ crossed, depth constancy for random-dot stereograms was present and single vision was reported. Outside this range, judgments were more variable but tended to fall around a fairly consistent level of near or far depth, regardless of the sign of the disparity. When viewing these large disparities, observers described the central region as being rectangular, with fuzzy edges, the component dots to be cloudy, "busy," or "swarming," and the depth to be nonuniform and unstable. ${ }^{1}$ On a small fraction of trials, large disparities were correctly reported in depth. On these trials, the central region was seen as square and stable, but now superimposed on a cloudy, busy, rectangular surround.

This reversal of depth sign is typically not reported with simple line stereograms; the phenomenon seems to be 
peculiar to random-dot stereograms (or, at least, stereograms with high contour density). In fact, depth estimates from line stereograms of the same dimensions and disparities tend to be veridical, whereas those from randomdot stereograms are relatively independent of disparity.

What causes large disparities to be seen sometimes in reversed depth? The tendency for the central region to appear rectangular rather than square suggests that observers are unable to match up dots in the disparate regions and, instead, respond to a region of interocularly uncorrelated dots. This idea is shown schematically in Figure 1.

To test this possibility, we constructed a variety of random-dot stereograms in which dots seen by the left and right eyes were perfectly correlated in one region (e.g., the surround) but were uncorrelated within another region (e.g., the center). We found that the entire uncorrelated area was seen as near or far depth, even though this region contained no consistent positional disparity. The following section describes observations bearing on this phenomenon of rivaldepth.

\section{Phenomenology}

Several interesting aspects of rivaldepth can be appreciated by stereoscopic inspection. Accordingly, some representative rivaldepth stereograms are given in Figure 2, so the reader may confirm most of the observations described below.

Inspection of a random-dot stereogram containing a central uncorrelated region with no overall horizontal disparity, such as Figure 2a, yields a sense of depth, the phenomenon we are calling rivaldepth. This percept is augmented when the observer views the region peripher-

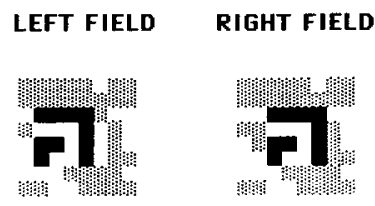

1 dot crossed dispority

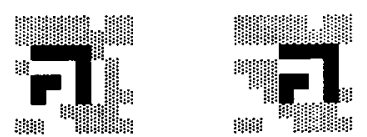

2 dots crossed disparity

\begin{tabular}{|l|r|}
\hline \multicolumn{1}{|c|}{ Key for half-images } & Key for bin. superimposition \\
Center dots & Correlated center dots \\
\hline Surround dots & Uncorrelated center dots \\
& Correlated surround dots \\
\hline
\end{tabular}

Figure 1. These drawings are meant to illustrate what happens when random-dot stereogram half-images are binocularty combined. Binocular correspondence can be established in random-dot stereograms when horizontal disparity is small (upper set of half-images) but not when disparity is large (lower set of half-images). The failure of correspondence places uncorrelated dots on corresponding retinal areas within the center of the stereogram.
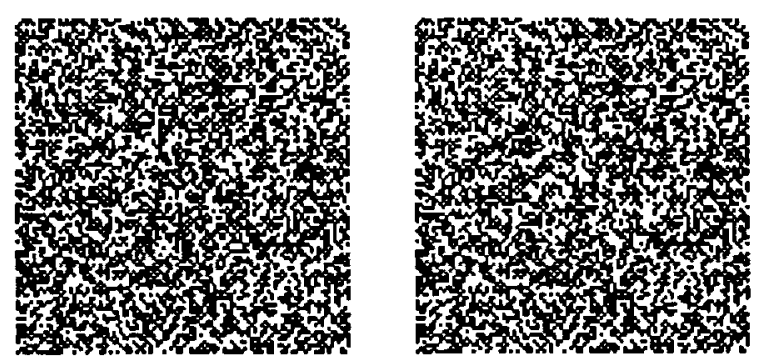

a
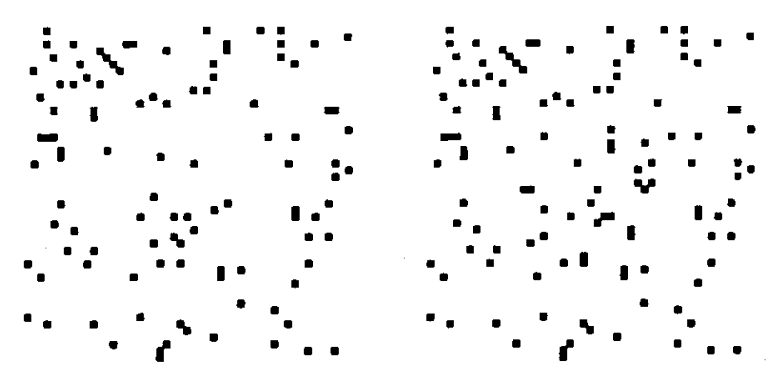

Figure 2. Demonstration rivaldepth stereograms. Rivaldepth will be experienced if these are binocularly combined, whether by stereoscope or with free fusion through overconvergence or divergence of the eyes. (a) This stereogram is a $100 \times 100$ matrix with a concentric square region of $\mathbf{4 0} \times \mathbf{4 0}$ dots. The surround is correlated, and the center is uncorrelated. Dot density is .5 . The central region should appear to be at a different depth from that of the surround; this is rivaldepth. Its direction (near or far) depends on individual differences. Compare this stereogram with that in Figure 3a to see that reducing the number of dots by a factor of four, while maintaining the same dot density, leaves rivaldepth unchanged. (b) This stereogram has the same dimensions of that in Figure 3a, but with a dot density of .005. Chance matches between individual dots are more noticeable, yet a global sense of rivaldepth remains, especially with peripheral viewing.

ally or squints. The boundaries of the central region may appear somewhat indistinct and, within them, individual dots may engage in binocular rivalry with their uncorrelated counterparts in the other half-image. On scrutiny, local regions may be seen at different depths up to or crossing the depth of the surround. These may fluctuate, but a global sense of depth that is relatively stable remains. ${ }^{2}$ Occasionally, the global sense of depth may reverse, although the predominant depth direction-near versus far-is easy to specify.

Rivaldepth seems to be relatively uninfluenced by the density of the dots making up the stereogram. This is illustrated in Figure 2b. So long as the dots are individually resolvable, rivaldepth is also unaffected by the size of dots. With very large dots, or when there are very few of them, rivaldepth is not so compelling, but rivalry between individual dots is evident (cf. Boman \& Kertesz, 1985). Rivaldepth is uninfluenced by luminance as long as the individual elements are visible. We have also observed rivaldepth with brief presentations (less than 
$100 \mathrm{msec}$ ) and in dynamic random-dot stereograms (presented at a frame rate of $22 \mathrm{~Hz}$ ), although in the latter case rivaldepth seemed more unstable than under static presentation (cf. Gonzalez, Krause, \& Poggio, 1986; Ross, 1974).

\section{EXPERIMENT 1}

Having explored phenomenologically some of the limits of rivaldepth, we set out to answer the following four specific questions: (1) Are there individual differences in preference for near and far rivaldepth? (2) Does rivaldepth reverse when the fields of stereograms are interchanged? (3) Is rivaldepth direction dependent on the nature of the rival contours contained in the stereograms? (4) Can the direction of rivaldepth be predicted from tests of binocular function? To answer these questions, data were obtained from a large sample of individuals.

\section{Method}

Subjects

Fifty-two unpaid volunteers participated in this study; all were naive to the phenomenon under study, although 13 had had some experience with viewing conventional random-dot stereograms. All subjects had monocular acuity sufficient to resolve the elements of each half-image of the stereograms, either without or with correction. All subjects could correctly report the form and depth in fully correlated random-dot stereograms with small crossed or uncrossed disparity.

Apparatus and Procedure. Eight rivaldepth stereograms of identical dimensions were drawn by an Apple Laserwriter, mounted on cardboard, and shown in a Holmes-type stereoscope. We created four different types of rivaldepth stereograms. Three contained fully correlated surrounds and the following types of interocular decorrelation in the center: (1) zero correlation (uncorrelation), (2) negative correlation (complementation), (3) patent rival targets (monocularly visible, orthogonal, diagonal contours). The fourth stereogram had an uncorrelated surround and a correlated center. These are shown in Figure 3 (identified as version $L$ ); the remaining four stereograms (version $\mathrm{R}$, not illustrated) were the same except with the half-images reversed. For each stereogram, the surround was $50 \times 50$ dots and the central regions consisted of $20 \times 20$ dots or an equivalent central area. In all four stereograms, the central region occupied exactly the same position-overall it had neither horizontal nor vertjcal disparity. The visual angle of the entire matrix at the focal plane of the stereoscope lenses was $8.58^{\circ} .^{3}$

A subject was first administered Julesz's (1971, pp. 273-275) random-dot test of stereopsis. This began with a fully correlated stereogram (its visual angle was $14.13^{\circ}$ ) containing crossed disparity, followed by similar stereograms with increasing amounts of complementation. The subject was scored on the largest amount of decorrelation at which he/she could see the form and depth of the central square. The test was then repeated for uncrossed disparities.

Next the subject was shown the eight rivaldepth stereograms in random order. For each stereogram, the subject reported whether its central square area appeared in front of the surround or behind the surround; "don't-know" responses were allowed. The subject's spontaneous comments were recorded verbatim.

Finally, fixation disparity and phoria were measured in the stereoscope. The fixation disparity stereogram consisted of correlated surrounds of random dots with the central area blank $\left(3.44^{\circ}\right)$, except for a single, central, upper vertical line ( $4.5^{\prime}$ in length; all lines
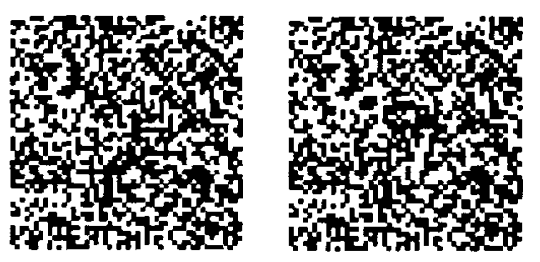

Center, Uncorr L

a
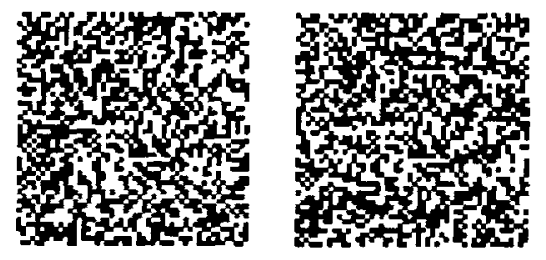

Surround, Uncorr $L$
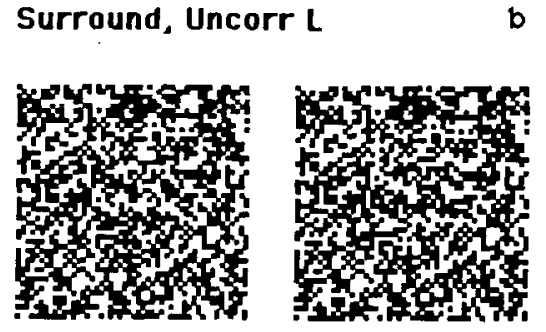

Center, Comp L

c
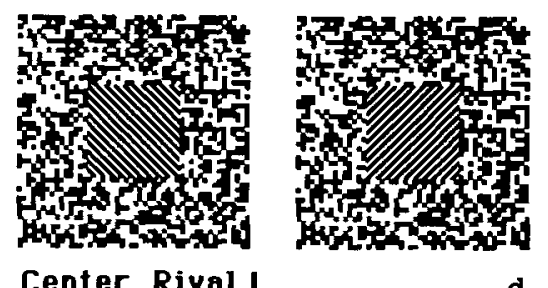

d

Figure 3. The four basic rivaldepth conditions of Experiment 1. (a) Uncorrelated center and correlated surround; the center should appear to be at a different depth from the surround. (b) Uncorrelated surround and correlated center; if a stable match in the center can be achieved, rivaldepth should be opposite to that observed above. (c) Complemented center and correlated surround; rivaldepth should be the same as in (a). (d) Rival center and correlated surround; the central region is replaced with dichoptically orthogonal diagonal lines, resulting in clear binocular rivalry. Nevertheless, rivaldepth in the same direction as in (a) should be observed.

were $0.5^{\prime}$ in width), presented to the left eye. Presented to the right eye was a series of equally spaced, numbered vertical lines, each separated horizontally from its neighbors by $1.5^{\prime}$; these were vertically separated from the single upper line by $3^{\prime}$. The subject was asked to read the number of the lower line that was aligned with the single upper line. The phoria targets consisted of a vertical arrow $\left(4.5^{\prime}\right)$ presented to the left eye and a horizontal scale ( 3 ' divisions) presented to the right. The subject was asked to read the number of the scale to which the arrow pointed.

Several weeks after the rivaldepth testing, 32 of the 52 subjects were recalled and presented with the anaglyphic version of Julesz's (1971, p. 350) ambiguous stereogram. It consists of a fully correlated surround, and a central square with a vertically repetitive pattern that provides equal probability matches for both a crossed 
and an uncrossed disparity. This was viewed through red-and-green glasses at reading distance $\left(40 \mathrm{~cm}\right.$ for a visual angle of $\left.12.50^{\circ}\right)$, first with the red filter over the right eye, then with the glasses reversed. Subjects were asked to say whether the central square region $\left(5.22^{\circ}\right)$ was nearer or farther than the surround.

\section{Results and Discussion}

Nearly all subjects responded quickly and confidently to the rivaldepth stereograms. Those who tarried reported depth fluctuations; they were asked to respond to the predominant depth. The research questions are answered below:

1. Individual differences in rivaldepth direction. Although uniform responses to the eight test stereograms (i.e., all near or all far) were given by only 5 subjects (fewer than $10 \%$ ), it is clear that subjects were not choosing directions by chance. Figure 4 shows the frequency distribution of preferences for far rivaldepth over the eight stereograms. The distribution is approximately rectangular; a chi-square test for goodness of fit to a binomial distribution (the distribution expected from chance responding) showed $\chi^{2}(7 ; n=42)=124.17, p<.001$, a significant departure from chance, and fit to a rectangular distribution was quite good $\left(\chi^{2}=7.71, p>.2\right)$. In other words, individual subjects generally preferred one direction of rivaldepth over the other. There is no obvious bias toward one particular direction of rivaldepth; across subjects, near rivaldepth was just as common as far depth.

2. Rivaldepth is unchanged by reversal of the halfimages. Product-moment correlations between rivaldepth responses to the two versions for each of the four types of stereograms should have been negative if depth had reversed when half-images were interchanged. In fact, all coefficients were positive: .82 (uncorrelated center), .51 (complemented center), .36 (rival center), and .52 (uncorrelated surround). This indicates that, unlike with conventional stereograms, rivaldepth tends to be in the same direction when the half-images are interchanged. Hence, rivaldepth cannot be explained on the basis of asymmetries between the eyes, the fields of the stereoscope, or the stereograms.

3. Rivaldepth direction is independent of the nature of the rival contours. Both hierarchical cluster analysis (see Thorndike, 1978) and factor analysis were used to classify stereograms on the basis of similarity of responses given to them. For the sake of brevity, results can be summarized as follows.

Stereograms with uncorrelated surrounds formed a group distinct from those with correlated surrounds. This grouping probably is related to the difficulty of judging depth in the uncorrelated surround stereograms. Upon viewing these stimuli, subjects frequently commented that the shape of the central region was difficult to discern. Inability to see the central square may have arisen from problems with binocular fixation. The relationship of fixation disparity to rivaldepth is considered below.

In general, cluster analysis and factor analysis show that rivaldepth direction is similar for various sorts of uncorre-

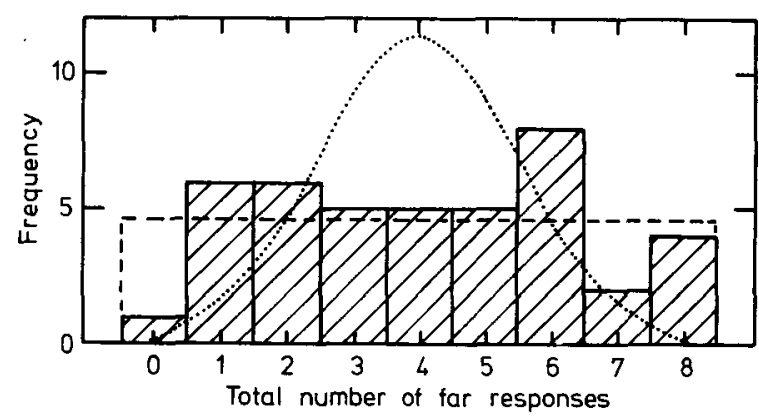

Figure 4. Frequency distribution of number of far-depth responses given by each subject to the eight stereograms. The dotted curve shows the distribution expected if subjects chose rivaldepth direction according to chance. The dashed curve shows the best-fitting rectangular distribution.

lation. Nonetheless, subjects' comments revealed notable differences among the various types of stereograms. In comparison with stereograms containing uncorrelated centers: complemented figures showed larger depth with more depth reversals; rival figures showed smaller depth and were harder to judge, and line orientation alternated; and those with uncorrelated surrounds were the hardest to judge, with subjects often discerning no central region in depth, or only an ill-defined, circular region.

4. Rivaldepth is related to fixation disparity. The several tests of binocular function showed the expected intercorrelations: for example, performance on the crossed-disparity stereograms of Julesz's test was highly related to performance on uncrossed-disparity stereograms $(r=.79)$. Fixation disparity and phoria were also highly related $(r=.74)$. Multiple regression was used to find a linear composite of binocularity measures that would predict rivaldepth direction. To this end, various composite scores of rivaldepth were generated, including factor scores from the factor analysis mentioned above. There were no essential differences between the degree to which any of the scores were predicted, so the correlations reported below are on the total number of far-depth responses out of the eight stereograms. Also, combining the binocularity measures did not improve prediction; stepwise regressions generally settled on a single predictor. The best predictor was fixation disparity, which showed a significant negative correlation [r(39) $=-.37, p<.05]$, such that divergences were associated with reports of near rivaldepth and convergences were associated with far depth (cf. Shippman \& Cohen, 1983). The remaining variables-stereopsis with decorrelation, phoria, and responses to Julesz's ambiguous stereogram-were minimally related to rivaldepth.

So, the results from this survey, besides documenting the robustness of the phenomenon, raise two interesting points about rivaldepth. First, rivaldepth is observed with several different types of interocular decorrelation, suggesting that rivalry has a depth sign. To our knowledge, no modern theory of stereopsis has included such a tenet. ${ }^{4}$ The existence of rivaldepth implies that such a tenet should 
be given more consideration. Second, the direction of rivaldepth bears some relationship to fixation disparity. Below we develop an explanation of this relationship, although admit at the outset that it is quite speculative and, as yet, largely untested.

We start with the assumption that uncorrelation signals the presence of an object so far in front of or behind the plane of fixation (i.e., the horopter) as to be beyond the limit of binocular fusion. This is entirely consistent, of course, with the phenomenon that motivated us to perform the survey experiment (recall Figure 1). Why, though, do some subjects report far rivaldepth and others report near rivaldepth? Consider the case of divergent fixation disparity associated with the percept of near rivaldepth (the argument is the same, except reversed, for the case of a convergence). Perhaps a given individual habitually executes a divergence when confronted with a binocular correspondence problem (cf. Julesz, 1971, p. 200). A divergence moves the fusion limits farther from the eyes. Since the problem is not solved by this manoeuver (i.e., the uncorrelated region is still uncorrelated), the visual system is biased to assume that the object was in the opposite direction, that is, near. This bias must override the information provided by the failure to establish binocular correspondence with subsequent convergent eye movements.

\section{EXPERIMENT 2}

There are a number of limitations of the survey method used in Experiment 1. For one, each stereogram was presented only once, so there was no opportunity to judge whether subjects were consistent in their reports to the same rivaldepth conditions. For another, responses were gathered only on rivaldepth direction, but, from spontaneous comments, there were indications that rivaldepth magnitude depended on type of uncorrelation (i.e., complementation was described by some subjects as appearing farther from the surround than uncorrelation). Also, Julesz and Tyler (1976) demonstrated perceptual differences between uncorrelation and complementation, in particular in the ease with which transitions between the two states were detected.

Experiment 2 was designed to overcome these limitations. Also, Experiment 2 allowed a closer simulation of the correspondence problem that occurs when one views correlated stereograms with large horizontal disparities. To this end, uncorrelated centers were shifted horizontally in each half-image by the same amount as that contained in stereograms with correlated centers. Below we will refer to this monocular shift of an uncorrelated area as "disparity," although strictly speaking this term is a misnomer. The leading edge of a horizontally shifted area obscures some potentially matchable dots in the surround, and its trailing edge "uncovers" some unmatchable dots. When the shifted area is uncorrelated to start with, horizontally shifting it simply has the effect of broadening the horizontal extent of uncorrelation. Nevertheless, it seemed possible that this manipulation might influence depth judgments. ${ }^{6}$

Manipulating disparity, while measuring depth magnitude, allows a test of the first assumption about the association between rivaldepth and fixation disparity. Specifically, the magnitude of rivaldepth from uncorrelation should be the same as depth from correlated stereograms with disparities beyond the fusion limit.

The ideal test of the second element of the relationship between fixation disparity and rivaldepth would involve objective measurement of eye position during inspection of a rivaldepth stereogram. As in the above example, an initial divergence might be followed by a convergence and the subject would report near rivaldepth. Since apparatus for measuring binocular eye movements was unavailable, we assessed eye position subjectively using nonius lines. Two tests were made: one test, which we shall call "tonic" fixation disparity, was a general, once-only measure similar to that made in Experiment 1; the other, which we shall call "phasic" fixation disparity, involved responses to nonius lines that appeared immediately after offset of each stereogram.

With these considerations in mind, Experiment 2 was designed to test a number of research questions: (1) Does horizontal disparity (i.e., enlarging the horizontal extent of uncorrelation) have any effect on rivaldepth or fixation disparity, in comparison to its effects on correlated stereograms? (2) Can naive subjects respond consistently to rivaldepth stereograms? (3) Are there differences in rivaldepth observed from uncorrelation and complementation? (4) What is the relationship between fixation disparity (both tonic and phasic) and rivaldepth?

\section{Method}

Subjects. Five female and 3 male students participated in this experiment in partial fulfillment of a course requirement. All had monocular uncorrected or corrected Snellen acuities of $6 / 6$ or better in each eye, with the exception of 1 male, who had uncorrected anisometropia (6/60 in his left eye); this individual wore no correction during testing. Each student possessed good stereopsis, as measured on the Titmus test of the Orthorater. All participants were naive as to the purposes of the experiment and the phenomenon of random-dot stereopsis. The point test of sighting dominance showed that each subject was right-eye dominant. Near and far phorias were within normal limits.

Apparatus. The subject's head was stabilized with a foreheadand chinrest, so that the screen of a Macintosh microcomputer was visible through a mirror stereoscope constructed from optical bench components. The Macintosh's mouse was used to register responses.

The random-dot stereograms were made up of an approximately square matrix $\left(6.53^{\circ}\right.$ horizontally $\times 6.68^{\circ}$ vertically; visual angles given below are for horizontal extents only), consisting of 100 $\times 100$ approximately square elements, each of which had an equal probability of being black or white. Each element subtended a visual angle of $3.92^{\prime}$. The stereograms contained a concentric central disparate region of $25 \times 25$ elements $\left(1.63^{\circ}\right)$. This area had a range of disparities added to it by shifting its horizontal position in equal and opposite directions in the two eyes and in integer multiples of one element. The range was from 16 elements of uncrossed disparity $\left(1.04^{\circ}\right.$; negative by our convention), through zero to 16 elements of crossed (positive by our convention). Stereograms were 
displayed on a half-tone field with white dots of $1.97^{\prime}$ in diameter (this will be referred to as gray).

Tonic fixation disparity was measured with a pair of nonius lines whose height and width were $40^{\prime} \times 4^{\prime}$; their separation was $10^{\prime}$. Concentric with the center of the gap between the nonius lines were annuli of $4^{\prime}$ width and internal radii of $0.82^{\circ}, 1.38^{\circ}$, or $2.45^{\circ}$; these were presented to both eyes. The upper nonius line was visible to the right eye and was fixed on the screen. The lower nonius line was visible to the left eye, and its initial position was in geometric alignment with the upper. The subject could vary the horizontal position of the lower nonius line by making horizontal movements with the mouse. The position of the lower line was sampled 1,200 times in $40 \mathrm{sec}$. All stimuli were black on a white screen.

To measure phasic fixation disparity on each trial, stereograms were immediately followed by brief presentation (144 msec) of a field containing nonius lines. This field consisted of the gray background upon which were drawn black nonius lines, each of $31^{\prime}$ length $\times 5^{\prime}$ width, separated by $33^{\prime}$.

The other fields used in the experiment consisted of (1) a uniform gray field, and (2) a response field that contained a Macintosh dialog box visible only to the left eye. The response dialog box consisted of the following: a schematic plan of the stereoscopic percept (the subject could move its central part by moving the mouse to estimate the depth experienced), and "buttons" to register a decision about the position of the nonius lines and to start the next trial.

Luminances were measured through the eyepiece with a Minolta spot photometer. Dark parts had a luminance of $0.27 \mathrm{~cd} / \mathrm{m}^{2}$, bright parts were $15.76 \mathrm{~cd} / \mathrm{m}^{2}$, and the gray had a space average luminance of $6.03 \mathrm{~cd} / \mathrm{m}^{2}$. Testing took place in a sound-attenuated, light-tight booth with dim indirect lighting such that the body of the Macintosh reflected $0.48 \mathrm{~cd} / \mathrm{m}^{2}$.

Procedure. To begin, the subject viewed nonius lines and adjusted the mirrors of the stereascope to correct for any phoria. Then tonic fixation disparity was measured: the subject kept the lower nonius line aligned with the upper by varying horizontal position of the lower line with the mouse. Trials lasted about $60 \mathrm{sec}$ and were separated by at least $60 \mathrm{sec}$. Each of the three surround radii was tested twice; the six trials were administered in a random order.

Next, depth and phasic disparity were measured. Each trial began with the appearance of nonius lines and the computer's emitting a beep. On a press of the mouse button, the stereogram was displayed. The subject was allowed free eye movements and as much time as required to estimate the depth of the central region relative to the surround. Once that judgment was made, the subject fixated the center of the stereogram and gave a buttonpress. This removed the stereogram and replaced it with the briefly presented nonius lines. The subject then used the dialog box to indicate whether the top nonius line was to the left or right of the lower. The final mouse click in the box ended the trial. Intertrial interval was 7 séc, during which the next stereogram was generated and the subject viewed a gray field.

The subjects responded to the full factorial combination of disparities (at least 9) and correlations (3), repeated 4 times in completely random order (at least 108 trials) in one session. One subject (M.R.B.) returned for two more sessions so that more repetitions and tests of new disparities could be made.

\section{Results and Discussion}

Subjects generally responded quickly and confidently to the uncorrelated stereograms, usually viewing them for about $1 \mathrm{sec}$ before making a response. The results of the anisometropic subject were not exceptional. The research questions posed will be dealt with in turn.

1. Rivaldepth is equivalent to depth from large disparities. Depth responses of one representative subject to complemented, uncorrelated, and fully correlated stereograms are shown in Figure 5. Notice first the clear relationship between perceived depth and physical disparity for the fully correlated stereograms. This clear trend is evident for all subjects, and merely confirms that the magnitude of perceived depth grows with disparity. Critically, however, at disparities around $1^{\circ}$, rivaldepth is observed, as evidenced by the change in the magnitude of the depth percept. For the stereograms with uncorrelated and complemented centers, all "disparities" produced about the same magnitude of depth which, for this subject, was seen as a rivaling central region situated behind a fused surround.

Note that mean depth responses and their standard errors have been computed for the uncorrelated and complemented stereograms. This is somewhat inappropriate, since these statistics are influenced by an underlying bimodality of responses (see below). Nevertheless, any systematic shift of depth direction related to disparity (i.e., horizontal extent) would be evident in the means. No effect of disparity was seen for this or any other subject.

Absolute depth responses (i.e., ignoring depth direction) were averaged at the largest disparity $\left(1.04^{\circ}\right)$ for the three types of correlation; averaging near- and fardepth absolute values eliminates the bimodality of responses. These data were analyzed by a two-factor, within-subjects analysis of variance. This analysis showed depth to be equivalent for the three types of stereograms $[F(2,14)=3.58, p>.05]$; also there were no differ-

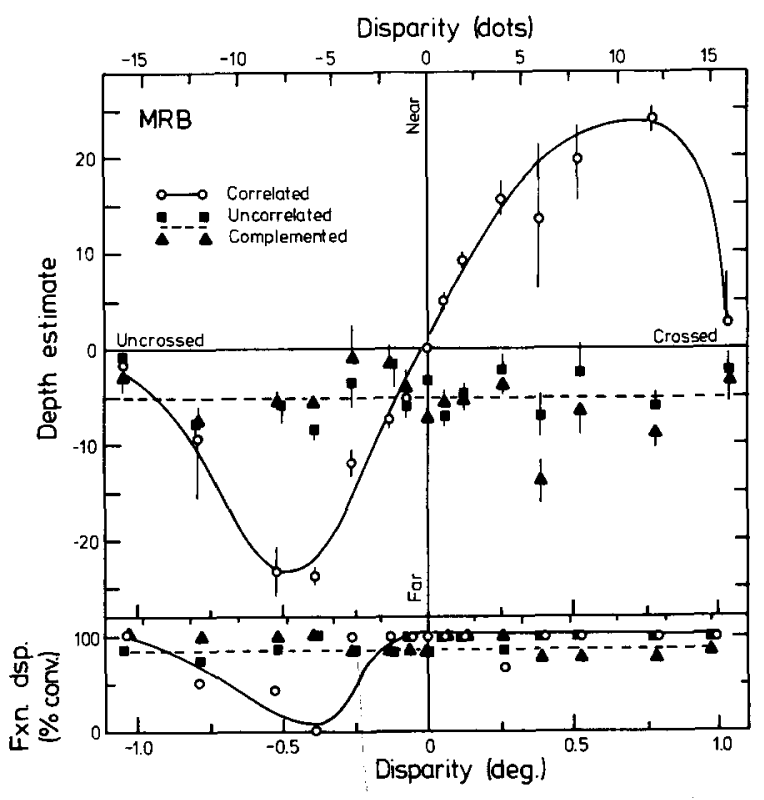

Figure 5. In the upper panel are plotted one subject's mean depth responses (arbitrary units) to correlated stereograms, and stereograms with uncorrelated and complemented centers, as a function of disparity. Vertical bars show standard errors. The lower panel shows phasic fixation disparity (the percentage of convergences) exhibited immediately following inspection of the various types of stereogram, plotted as a function of stereogram disparity. 
ences between crossed and uncrossed disparities $(F=$ 0.22 ) and no significant interaction between type of stereogram and disparity $(F=0.36)$. The statistical analysis confirms what is evident in Figure 5: rivaldepth is of the same magnitude as depth from correlated stereograms with large disparity. Thus, our first assumption about the relationship between fixation disparity and rivaldepth is supported.

Correlated stereograms did lead to phasic vergence changes, as indicated in the lower panel of Figure 5 . As expected, crossed disparities were associated with convergent eye movements and uncrossed with divergent ones. For the rivaldepth stereograms, there were no significant effects of disparity or type of complementation on fixation disparity for this or any other subject. Eye movements in response to rivaldepth stereograms are considered further below.

2. Rivaldepth is intrinsically variable. Since horizontal extent did not affect rivaldepth, responses were cumulated over all "disparities." All subjects except 2 showed an asymmetric, bimodal distribution of depth responses, with the trough between the two modes at zero depth. Seven subjects preferred far depth, and 1 preferred near depth. Even if depth direction is ignored, average standard deviation (expressed as a proportion of the mean) of rivaldepth responses was about 1.5 times greater than that for depth responses to fully correlated stereograms. The bimodal distribution of rivaldepth responses suggests that reversibility is intrinsic to rivaldepth stereograms.

3. Uncorrelation and complementation yield equivalent rivaldepth. In their signed rivaldepth responses, 7 subjects showed no difference between uncorrelation and complementation, measured over all stereograms. The remaining subject did show differences, for which we have no explanation. After all, complementation in stereograms containing disparity would require precise vergence changes to achieve the "goal" of complementary stimuli falling on corresponding retinal points. Examination of phasic fixation disparity showed that this subject did not make such eye movements.

The critical comparison is at zero disparity, where complementary stimuli $d o$ fall on corresponding retinal areas. The bimodal distributions of responses presented a problem for statistical comparison. Accordingly, sign was ignored and differences between depth responses to complemented and uncorrelated stereograms were assessed by $t$ tests for each individual and by a correlated $t$ test for the means of all subjects. No significant differences were found [e.g., the latter test found $t(7)=1.54$, $p>.1]$. There were also no differences in the pattern of phasic fixation disparities associated with uncorrelated and complemented stereograms $[t(7)=0.96]$.

4. Rivaldepth is related to fixation disparity. To assess the relationship between tonic fixation disparity and rivaldepth, a matrix of product-moment correlations was computed for all measures including near and far phorias. Rivaldepth was expressed for each subject as the mean of all signed rivaldepth responses. With only 8 subjects,
Table 1

Correlation Coefficients for the Relationship Between Phasic Fixation Disparity and Depth for Conventional (Fully Correlated) and Rivaldepth (Uncorrelated and Complemented) Random-Dot Stereograms

\begin{tabular}{lllll} 
& \multicolumn{3}{c}{ Stereogram Type } \\
\cline { 2 - 5 } Subject & \multicolumn{3}{c}{ Conventional } & \multicolumn{2}{c}{ Rivaldepth } \\
\hline M.R.B. & $0.52 \S$ & $(87)$ & $0.16^{*}$ & $(169)$ \\
S.L. & $0.59 \S$ & $(48)$ & $0.18^{*}$ & $(96)$ \\
L.S. & & $(36)$ & & $(72)$ \\
S.R. & $0.46 \ddagger$ & $(36)$ & $0.42 \S$ & $(72)$ \\
J.J. & $0.34 \dagger$ & $(36)$ & 0.12 & $(72)$ \\
D.H. & $0.44 \ddagger$ & $(36)$ & $0.22^{*}$ & $(72)$ \\
S.H. & $0.81 \S$ & $(31)$ & $0.60 \S$ & $(66)$ \\
D.F. & $0.58 \S$ & $(36)$ & $0.29 \ddagger$ & $(72)$ \\
\hline
\end{tabular}

Note-Numbers in parentheses are the number of observations. Values were indeterminant for Subject L.S. because of no variance in fixation disparity. ${ }^{*} p<.1 . \quad \neq p<.05 . \quad \ddagger p<.01 . \$ p<.001$.

such coefficients are suggestive rather than definitive. The critical relationship between tonic fixation disparity (for the smallest radius of surround) and rivaldepth was $r=-.42$, which is in the same direction as found in Experiment 1 . As would be expected, coefficients were high between the various measures of tonic fixation disparity $(r s$ around .95).

To quantify the relationship between phasic fixation disparity (i.e., that measured at stereogram offset) and rivaldepth, two Pearson product-moment coefficients were computed for each subject between signed depth response and vergence report. One was for correlated stereograms and the other was for rivaldepth (i.e., uncorrelated and complemented) stereograms. The results for each subject are presented in Table 1. The table shows the direct relationship between magnitude of depth and vergence for correlated stereograms; subjects made convergent eye movements when reporting near depth and divergent movements when reporting far depth. For rivaldepth stereograms, the correlations are smaller, but still positive. These positive correlations are in contrast to the negative correlation between tonic fixation disparity and rivaldepth.

The negative correlation between tonic fixation disparity and rivaldepth confirms that found in the survey. The positive relationship between phasic fixation disparity (i.e., eye position at rivaldepth stereogram offset) and rivaldepth adds a wrinkle to the second element of our explanation: It seems that subjects attempt to fuse the uncorrelated center by making vergences toward its apparent depth.

\section{GENERAL DISCUSSION}

There have been a number of reports of unexpected depth percepts from random-dot stereograms, of which some are probably instances of rivaldepth. For example, Julesz (1960) cites Wegner (1959) as finding that uncorrelated central dots are seen behind a correlated surround. Moreover, Julesz (1971, pp. 20 and 260) noted the complementary case: a correlated central region appearing in front of an uncorrelated surround. Frisby and Mayhew 
(1979) presented a random-dot stereogram containing a central uncorrelated region (as in Figure 3); they reported that perceived depth oscillated between near and far, and that its direction could be voluntarily controlled. Rivaldepth has possibly also been observed in stereograms consisting of a pair of short lines with a dichoptic orientation difference of $90^{\circ}$. Mitchell and O'Hagan (1972) found individual differences in the direction of disparity that had to be added to make the orthogonal pair appear at the same depth as a nonorthogonal reference pair.

There are other unexpected stereoscopic depth effects, which we believe should be distinguished from those on rivaldepth. Julesz (1964) observed that there were invariably vertical edges of uncorrelated dots immediately adjacent to a disparate area in a random-dot stereogram (see Figure 1). The width of these edges depends on the magnitude of the disparity, and the perceived depth of these regions tends to correspond to the plane of the farthest neighboring region (i.e., to the zero depth surround for a crossed-disparity center and to far depth for uncrossed disparity). As Julesz noted, such effects have a ready geometrical explanation as versions of Panum's limiting case. In fact, random-dot stereograms can be drawn with a single column of dots added to the left or right side of the left or right field, and they will be seen as near or far in depth according to the geometry of Panum's limiting case. Our zero-disparity rivaldepth stereograms, however, do not satisfy the stimulus conditions for Panum's limiting case, nor is the depth from such stereograms invariably associated with that of the farthest neighboring region.

Collet (1985) tested depth in random-dot stereograms in which certain areas were devoid of dots in one halfimage. He reported that the monocularly unmatched areas were seen in the depth plane of the adjacent region. These stimuli differ from rivaldepth stereograms in that they lack dichoptically overlapping contours, which we believe are necessary for rivaldepth.

Frisby and Mayhew (1978) conducted studies on stereograms that bear some resemblance to those reported here. Their "rivalrous texture stereograms" contained binocularly uncorrelated center and surround regions of different spatial frequencies within each half-image. They observed depth even with zero disparity, with the highspatial-frequency area appearing closer. There are, however, several notable differences between such rivalrous texture stereograms and ours. Since their stereograms had both uncorrelated center and surround, in our terms there was rivaldepth in both regions. As to what might differentiate rivaldepths in center and surround, Frisby and Mayhew observed that the near depth associated with highspatial-frequency regions was visible monocularly, a phenomenon that has been recently rediscovered within another context by Wong and Weisstein (1985). In comparison, no figure-ground segregation is evident from monocular viewing of either half-image of our stereograms.

Frisby and Mayhew (1978) also showed a stereogram in which a monocular central region of one spatial fre- quency on a binocularly correlated surround of a different spatial frequency was seen in depth despite there being no matching region in the other half-image. They called the stereoscopic experience "paradepth" (for paradoxical depth). The difference between it and rivaldepth is more than nominal: there are no monocularly visible cues to the position of the central region in rivaldepth stimuli; for paradepth, as with their rivalrous texture stereograms, areas of high spatial frequency appear monocularly as figure superimposed on a background of low spatial frequency. Paradepth depends on the horizontal extent of the region; rivaldepth is not so dependent. O'Shea (1983) has argued that paradepth is an example of depth from monocular stimuli; it shows the same characteristics as those detailed by Kaye (1978). In particular, paradepth reverses when the central region is presented to the other eye (this may be confirmed by inverting any of Frisby and Mayhew's paradepth stereograms); rivaldepth does not so reverse.

Is it surprising that rivaldepth occurs? It has long been known that depth can be perceived from stereograms composed of rival half-images (Helmholtz, 1909/1962; Mayhew \& Frisby, 1976; Ramachandran, Rao, Sriram, \& Vidyasagar, 1973). In such stereograms, however, figure is segregated from ground by spatial frequency differences, which thus carry the disparity information (Kaufman, 1974). Furthermore, Mayhew, Frisby, and Gale (1977) have shown that Marr and Poggio's (1976) stereo computation algorithm can find the correct disparate figure in such stereograms. With rivaldepth stereograms, however, positional disparity defines no global region, nor do spatial frequency differences delimit figure from ground. So it does seem surprising that uncorrelated regions yield global depth in the absence of horizontal disparity.

No modern theory of binocular vision accommodates the phenomenon of rivaldepth. Thus, we hope our observations will encourage theorists to consider the depth information provided by rival areas. There is, however, very recent electrophysiological data that may elucidate the neural concomitants of rivaldepth. Gonzalez et al. (1986) presented a variety of stereoscopic patterns over the receptive fields of binocularly activated neurons in cortical areas V1, V2, and V3 of alert rhesus monkeys. They found single cells in all areas that were excited by line stereograms with either crossed disparity or uncrossed disparity. Many of these near and far cells were also activated by dynamic random-dot stereograms containing areas of uncorrelation with no overall horizontal disparity. These cells may represent the physiological substrate for the psychophysical observations we have made.

While these experiments were in progress, we became aware of the work of Holliday and Braddick (1985). They required subjects to align nonius lines before flashing stereograms containing an area of uncorrelation. The stereograms were presented for a time too brief to allow eye movements, yet rivaldepth (not the term they used) was reported. Holliday and Braddick also imposed dis- 
parities on the adjoining correlated area, and they found that rivaldepth tended to be in the same direction as the depth in the correlated region. A comparable observation has been described by Julesz and Chang (1976).

This observation is consistent with our finding of a modest relationship between fixation disparity and rivaldepth direction. A divergent fixation disparity would produce crossed disparities in the initially zero-disparity correlated surround; hence, the rivaldepth appears near. However, Holliday and Braddick's findings also imply that fixation disparity is not necessary for rivaldepth.

Our observations extend those of Holliday and Braddick by showing rivaldepth with free inspection. Moreover, once fixation disparity effectively changes disparity of neighboring correlated regions, rivaldepth must be fairly resistant to subsequent eye movements. This conclusion follows from the results of Experiment 2, which show that subjects attempt to fuse the uncorrelated region and thus tend to reverse the effective correlated disparity and, hence, the rivaldepth. We should note that such vergence changes may account for the alternations in rivaldepth that subjects have reported with prolonged inspection.

In summary, depth is observed in random-dot stereograms that have disparities beyond the limits of fusion. The direction of such depth is generally consistent within individuals. The depth is the same for zero-disparity decorrelated areas, suggesting that the large-disparity correlated case involves a failure to match the potentially correlated dots. We have called this rivaldepth to emphasize that the stimulus conditions for rivalry are required; in simple line stereograms with large disparities, neither rivalry nor rivaldepth is observed. Rivaldepth is stereoscopic, it is cyclopean, and it is related to fixation disparity. Rivaldepth is different from conventional stereoscopic vision in that depth does not reverse when the half-images are interchanged. An area showing rivaldepth seems to be treated as a potentially fusable surface beyond the fusion limit.

\section{REFERENCES}

Boman, D. K., \& KerTESZ, A. E. (1985). Effect of stimulus parameters on fusional and stereoscopic performance. American Journal of $O p$ tometry \& Physiological Optics, 62, 222-227.

CoLLETT, T. S. (1985). Extrapolating and interpolating surfaces in depth. Proceedings of the Royal Society of London, Series B, Biological Sciences, 224, 43-56.

FrisbY, J. P., \& MAYHEW, J. E. W. (1978). The relationship between apparent depth and disparity in rivalrous-texture stereograms. Perception, 7, 661-678.

FrisBY, J. P., \& MAYHEW, J. E. W. (1979). Depth inversion in randomdot stereograms. Perception, 8, 397-399.

Gonzalez, F., Krause, F., \& Poggio, G. F. (1986). Sensitivity of cortical visual neurons to binocular correlation of dynamic randomdot stereo patterns. Investigative Ophthalmology \& Visual Science, 27(Suppl.), 244.

HELMHOLTZ, H. L. F. vON (1962). Treatise on physiological optics (J. P. C. Southall, Trans.). New York: Dover. (Original work published 1909)

HOCHBERG, J. (1964). Contralateral suppressive fields of binocular combination. Psychonomic Science, 1, 157-158.
Holliday, I. E., Braddick, O. J. (1985). Depth perception of uncorrelated areas in random-dot stereograms. Perception, 14, A25-26. JuLESz, B. (1960). Binocular depth perception of computer-generated patterns. Bell System Technical Joumal, 29, 1125-1162.

JULESZ, B. (1964). Binocular depth perception without familiarity cues. Science, 145, 356-362.

Julesz, B. (1971). Foundations of cyclopean perception. Chicago: University of Chicago Press.

Julesz, B., CHANG, J. (1976). Interaction between pools of binocular disparity detectors tuned to different disparities. Biological Cybernetics, 22, 107-119.

JulESZ, B., TYLER, C. W. (1976). Neurontropy, an entropy-like measure of neural correlation in binocular fusion and rivalry. Biological Cybernetics, 23, 25-32.

Kaufman, L. (1974). Sight and mind: An introduction to visual perception. New York: Oxford University Press.

KAYE, M. (1978). Stereopsis without binocular correlation. Vision Research, 18, 1013-1022.

MARR, D., PoGG10, T. (1976). Cooperative computation of stereo disparity. Science, 194, 283-287.

MAYHeW, J. E. W., \& FrisBY, J. P. (1976). Rivalrous texture stereograms. Nature, 264, 53-56.

Mayhew, J. E. W., Frisby, J. P., \& Gale, P. (1977). Computation of stereo disparity from rivalrous texture stereograms. Perception, 6, 207-208.

Mitchell, D. E., \& O'HaGan, S. (1972). Accuracy of stereoscopic localization of small line segments that differ in size or orientation for the two eyes. Vision Research, 12, 437-454.

O'SHEA, R. P. (1983). Spatial and temporal determinants of binocular contour rivalry. Unpublished doctoral dissertation, University of Queensland, Australia.

Ramachandran, V. S., Rao, V. M., Sriram, S., Vidyasagar, T. R. (1973). The role of colour perception and pattern recognition in stereopsis. Vision Research, 13, 505-509.

RicHARDS, W. (1977). Stereopsis with and without monocular contours. Vision Research, 17, 967-969.

Ross, J. (1974). Stereopsis by binocular delay. Nature, 248, 363-364. SHIPPMAN, S., \& COHEN, K. R. (1983). Relationship of heterophoria to stereopsis. Archives of Ophthalmology, 101, 609-610.

THORNDIKE, R. M. (1978). Correlational procedures for research. New York: Gardner Press.

WEGNER, K. (1959). Stereoskopische Untersuchungen fur Tiefenlokalisation sogenannter funktionsloser Bestandteile des binocularen Gesichtsfeldes. Unpublished thesis, Georg August University, Göttingen, Germany.

WheAtstone, C. (1838). Contributions to the physiology of vision.Part the first. On some remarkable, and hitherto unobserved, phenomena of binocular vision. Proceedings of the Royal Society of London, Series B, 18, 371-394.

Wong, E., \& WeISsTeIn, N. (1985). Spatial frequency affects figureground organization. Investigative Ophthalmology \& Visual Science, 26(Suppl.), 281.

\section{NOTES}

1. There have been at least two similar findings. Richards (1977) briefly flashed random-dot stereograms, and found that veridical depth reports broke down above a certain disparity, to become either near or far depth whose magnitude was unrelated to disparity. Frisby and Mayhew (1979) reported that they could achieve a voluntary reversal of the depth of small-disparity random-dot stereograms by making the appropriate vergences. The reversed depth plane had the same rivaling appearance we note.

2. It is not strictly true that there is no disparity in stereograms leading to rivaldepth: local regions of depth could arise from disparity produced by chance matches of dots within the uncorrelated region. However, the average disparity is zero, and a global percept of depth should therefore be impossible. Yet the rivaldepth depth percept is global, in that the entire uncorrelated region lies at essentially the same depth plane. Moreover, a zero-disparity, uncorrelated region can be seen in depth when that region is composed of pattern elements for which even 
local matches are impossible. This can be seen in Figure 3d, where the central regions are composed of orthogonally oriented lines-the rivaling region appears to lie at a different depth from that of the textured surround even though there is no positional disparity in the stereogram.

3. Subjects chose their own viewing distances in the stereoscope, so actual visual angles could be as small as $7.00^{\circ}$ or as large as $14.50^{\circ}$. Most, however, placed the stereograms at or near the focal plane; subsequent visual angles will be given for the focal plane. The lenses of the stereoscope ensured that the stereograms were presented at optical infinity.

4. This assertion includes suppression theories of binocular vision (see Kaufman, 1974). These employ rivalry to eliminate diplopia, but stereopsis is generally accomplished by other mechanisms. The exception is Hochberg's (1964) proposal that both single vision and stereopsis were accomplished by rivalry. The rivalry was different from what is commonly understood by the term: the rival areas were small, forming a mosaic of left- and right-eye views. Stereopsis was accomplished by vernier-type discriminations of offsets between the small areas of dominance, requiring utrocular information. That is, "rivalry" was be- tween similar stimuli within Panum's fusional areas. The rivalry in the present stereograms was of the conventional type, between dissimilar stimuli.

5. Of course, in correspondence problems that do have a solution (i.e., stereograms containing crossed disparities), subsequent convergences would find the match, and so the first, unsatisfactory, guess would be ignored.

6. Frisby and Mayhew (1978) showed that horizontal extent affected a phenomenon similar to rivaldepth, namely paradepth. Their stereograms were similar to ours, except that one entire half-image and the surround of the other were spatially filtered in one way (e.g., low-pass filtered) and the center of the other was filtered in the opposite way (i.e., high-pass filtered). Since horizontal shifts of uncorrelated regions simulate horizontal disparity, it may be that rivaldepth would be affected by the horizontal extent of uncorrelation.

(Manuscript received August 25, 1986; revision accepted for publication March 26, 1987.) 